or otherwise promote their assembly into a three-dimensional lattice (Fig. 2b). Likewise, from this report it appears that the desmoplakin $\mathrm{N}$-terminal domain may also position plakophilin favourably to connect to the calyx of keratin heads projecting from intermediate filaments running parallel to the cell membrane. Given the ability of plakophilin to bind both actin and intermediate filaments, it could provide cellular resilience by coordinating both elements of the cortical cytoskeleton while the desmoplakin Cterminal domain connects to cross-bracing intermediate filaments approaching from a perpendicular route.

The in vitro rescue studies described by Vasioukhin et al. predict that in vivo knock-in of the desmoplakin N-terminal domain should restore a significant proportion of desmosomal and complexus adherens junction function, and may be compatible with life. Indeed, some clues are already available from human mutations. The phenotypes associated with null mutations of plakophilin1, C-terminal truncation of plakoglobin in Naxos disease, and the haploinsufficiency of desmoplakin support the essential role of these proteins in desmosomal structure ${ }^{2,5,21}$. A mutation resulting in C-terminal truncation of desmoplakin has also been described that is recessive and causes heart, hair and skin defects very similar to those produced by the plakoglobin mutation in Naxos disease $^{1}$. This desmoplakin mutation is, however, most significant for the phenotypes that it does not produce, allowing us to conclude that the cytoskeletal-binding function of desmoplakin is of minor importance in many epithelial tissues and without consequence for the vasculature. Taken together with the incongruence between the plakoglobin- and desmoplakin-null phenotypes in the vasculature, this suggests that the desmoplakin $\mathrm{N}$ terminus must engage in plakoglobin-independent linkages that cluster VE-cadherin in the endothelial complexus adherens junction, perhaps similar to those found in the desmosome. We look forward to further instalments in this saga, in which knock-in mice and cells from humans with desmoplakin mutations will contribute to elucidating the molecular mechanism of these cell-cell junctions and their role in cortical actin remodelling.
Sarah Hatsell and Pamela Cowin are in the Departments of Cell Biology and Dermatology, New York University School of Medicine,

550 First Ave, New York, New York 10016, USA email: hatses01@med.nyu.edu

email:cowinp01@med.nyu.edu

1. Norgett, E. E. et al. Hum. Mol. Genet. 9, 2761-2766 (2000)

2. McKoy, G. et al. Lancet 355, 2119-2124 (2000).

3. Ruiz, P. et al. J. Cell Biol. 135, 215-225 (1996)

4. Bierkamp, C., McLaughlin, K. L., Schwarz, H., Huber, O. \& Kemler, R. Dev. Biol. 180, 780-785 (1996).

5. Armstrong, D. K. et al. Hum. Mol. Genet. 8, 143-148 (1999).

6. Vasioukhin. V. et al. Nature Cell Biol. 3, 1076-1085 (2001).

7. Mathur, M., Goodwin, L. \& Cowin, P. J. Biol. Chem. 269, 14075-14080 (1994).

8. Kowalczyk, A. P. et al. J. Cell Biol. 139, 773-784 (1997).

9. Klymkowsky, M. W. Nature Cell Biol. 1, E121-E123 (1999).

10. Stappenbeck, T. S. \& Green, K. J. J. Cell Biol. 116, 1197-1209 (1992).

11. Smith, E. A. \& Fuchs, E. J. Cell Biol. 141, 1229-1241 (1998).

12. Kowalczyk, A. P. et al. J. Cell Sci. 111, 3045-3057 (1998).

13. Kowalczyk, A. P. et al. J. Biol. Chem. 274, 18145-18148 (1999).

14. Hatzfeld, M., Haffner, C., Schulze, K. \& Vinzens, U. J. Cell Biol. $149,209-222$ (2000)

15. Gallicano, G. I. et al. J. Cell Biol. 143, 2009-2022 (1998).

16. Gallicano, G. I., Bauer, C. \& Fuchs, E. Development 128, 929-941. (2001).

17. Gumbiner, B., Stevenson, B. \& Grimaldi, A. J. Cell Biol. 107, 1575-1587 (1988).

18. Adams, C. L., Nelson, W. J. \& Smith, S. J. J. Cell Biol. 135, 1899-1911 (1996).

19. Angres, B., Barth, A. \& Nelson, W. J. J. Cell Biol. 134, 549-557 (1996).

20. Vasioukhin, V., Bauer, C., Yin, M. \& Fuchs, E. Cell 100, 209-219 (2000).

21. McGrath, J. A. et al. Nature Genet. 17, 240-244 (1997).

\title{
Natural sunscreen revealed
}

The exposure of skin to ultraviolet B (UVB) radiation has a destructive effect on keratinocytes by causing DNA damage that can subsequently lead to malignant transformation. Cellular defence mechanisms against this injury begin with the immediate UVB-induced death of damaged cells. In less severely affected cells, cell-cycle progression can be blocked and DNA damage can be repaired through nucleotideexcision repair (NER). If repair is successful and cells have not suffered irreversible damage, they will survive. In the absence of appropriate repair, keratinocytes undergo apoptosis, producing characteristic 'sunburn cells'. In a recent advance online publication article in Nature Cell Biology, DOI: 10.1038/ncb717, Schwarz and colleagues provide evidence for a new participant in the DNA-damage response to UVB - the cytokine interleukin-12 (IL-12).

IL-12 is known primarily for its crucial role in the immune system. Now we have evidence that it can also enhance DNA repair in response to UVB. Schwarz and colleagues examined keratinocytes exposed to UVB in vitro and found that IL-12 can reduce apoptosis and improve their survival. This effect only applies to UVB, as IL-12 gives no resistance to $\gamma$-irradiation. Moreover, when mice themselves were exposed to radiation, IL12 knockout mice produced greater numbers of sunburn cells than did wild-type mice, a phenotype that is partially restored by subcutaneous injection of IL- 12 .

The authors report that treatment with IL-12, both in vitro and in vivo, is associated with a reduction in pyrimidine dimers, the predominant form of UVB-induced DNA damage. No reduction is seen 10 minutes after UVB irradiation, but is apparent 3.5 hours later, implying that IL-12 is having an effect following the initial insult. A mechanistic explanation is suggested

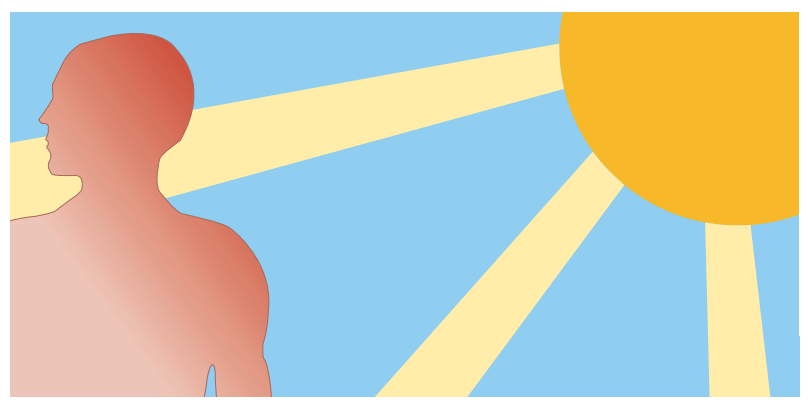

by their observation that IL-12 treatment enhances NER and is associated with increased expression of NER-associated genes. As further confirmation of this hypothesis, IL-12 affords no protection to Xpa knockout mice, which lack a component of the NER system.

These findings place IL-12 in a unique position of protecting against UVB by enhancing DNA repair, apparently without affecting apoptosis of damaged cells. Details of this process remain to be elucidated, as well as determining whether other cytokines might also modulate NER. Despite the unknowns, this report makes one speculate on the possibility of using IL-12 to protect against DNA mutations that can lead to skin cancer. In addition, deficiencies in IL-12 production in response to UVB might predispose humans to such cancers.

\section{SAQUIB LAKHANI and RICHARD A. FLAVELL Yale University School of Medicine e-mail: Richard.Flavell@yale.edu}

